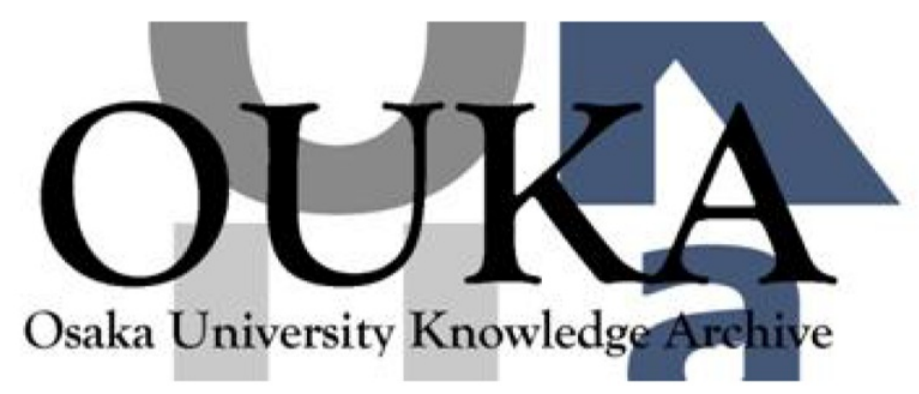

\begin{tabular}{|c|l|}
\hline Title & $\begin{array}{l}\text { Parallel distributed processing neuroimaging in } \\
\text { the Stroop task using spatially filtered } \\
\text { magnetoencephalography analysis }\end{array}$ \\
\hline Author(s) & Ukai, Satoshi \\
\hline Citation & \\
\hline Issue Date & \\
\hline oaire:version & VoR \\
\hline URL & https://hdl. handle. net/11094/44611 \\
\hline rights & \\
\hline Note & \\
\hline
\end{tabular}

Osaka University Knowledge Archive : OUKA

https://ir. Library. osaka-u. ac. jp/

Osaka University 


\title{
Parallel distributed processing neuroimaging in the Stroop task using spatially filtered magnetoencephalography analysis
}

\author{
Satoshi Ukai ${ }^{a}{ }^{*}$, Kazuhiro Shinosaki ${ }^{a}$, Ryouhei Ishii ${ }^{a}$, Asao Ogawa ${ }^{a}$, \\ Yuko Mizuno-Matsumoto ${ }^{a}$, Tsuyoshi Inouye ${ }^{a}$, Norio Hirabuki ${ }^{\mathrm{b}}$, Toshiki Yoshimine ${ }^{\mathrm{c}}$,

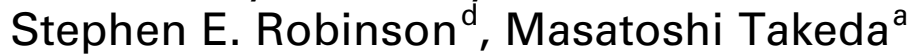 \\ ${ }^{a}$ Department of Psychiatry and Behavioral Science, Osaka University Graduate School of Medicine, D3, 2-2, Yamada-oka, \\ Suita City, Osaka, 565-0871 Japan \\ ${ }^{b}$ Department of Radiology, Osaka University Graduate School of Medicine, D3, 2-2, Yamada-oka, Suita City, Osaka, 565-0871 Japan \\ ${ }^{c}$ Department of Neurosurgery, Osaka University Graduate School of Medicine, D3, 2-2, Yamada-oka, Suita City, Osaka, 565-0871 Japan \\ ${ }^{d}$ CTF Systems, Inc., Port Coquitlam, British Columbia, Canada
}

Received 22 July 2002; received in revised form 26 August 2002; accepted 1 September 2002

\begin{abstract}
Parallel distributed processing neuroimaging in the Stroop color word interference task in five healthy subjects was studied. The total reaction time was set at $650 \mathrm{~ms}$ with a time window of $200 \mathrm{~ms}$ in steps of $50 \mathrm{~ms}$. Spatially filtered magnetoencephalography analysis, as used in synthetic aperture magnetometry, was used. Neural activation began in the left posterior parietal-occipital area (150-250 ms post-stimulus), followed by the right prefrontal polar area (250-350 $\mathrm{ms})$, the left dorsolateral prefrontal cortex $(250-400 \mathrm{~ms})$, and the mid- to lower- primary motor area $(350-400 \mathrm{~ms})$. Successive and temporally overlapping activation of various cortical regions were successfully estimated within a short $200 \mathrm{~ms}$ time interval, contrary to previous positron emission tomography and fMRI studies.
\end{abstract}

(C) 2002 Elsevier Science Ireland Ltd. All rights reserved.

Keywords: Dorsolateral prefrontal cortex; Inhibition; Magnetoencephalography; Neural network; Neuroimaging; Parallel distributed processing; Spatial filter; Stroop task

The Stroop color word interference task is utilized to assess frontal lobe functions [6,14]. Previous lesion studies demonstrated functional involvement of the left dorsolateral prefrontal cortex (DLPFC) [15] and left/right lateral frontal regions $[9,17]$ in this task. Positron emission tomography (PET) and fMRI studies have consistently showed increased activity in the anterior cingulate cortex (ACC) $[1,2,8,10]$ with one recent study demonstrating it in the left DLPFC [5]. The dissociation between the neuropsychological and the neuroimaging approaches is thought to be due to parallel distributed processing (PDP) in multiple cortical regions, subserving the sequential information processing from perception, attention, read inhibition and vocalization which are temporally overlapping. Time intervals of 100 ms are necessary for PDP visualization, since reaction times are approximately $650 \mathrm{~ms}$. PET functional activity

\footnotetext{
* Corresponding author.Tel.: +81-6-6879-3051; fax: +81-66879-3059

E-mail address: ukai@psy.med.osaka-u.ac.jp (S. Ukai).
}

could not be measured to better than $10 \mathrm{~s}$ and event-related fMRI studies of whole brain were measured within a 1650 ms cycle.

Magnetoencephalography (MEG) analysis can directly probe neural activity within a few milliseconds. The equivalent current dipole (ECD) MEG analysis is often used, but this method is only valid for discrete small spatial extent sources. Synthetic aperture magnetometry (SAM) is a novel spatial filtering technique based on the nonlinear constrained minimum-variance beam former [11]. This technique can estimate unambiguous three-dimensional source mapping. Taniguchi et al. reported the relative colocalization of event-related desynchronization (ERD) of the mu rhythm estimated by SAM with ECD and fMRI activation [16]. Using SAM, we have previously reported the frontal midline theta rhythm related focused attention task was generated in ACC [3]. In these studies, steady states were assumed to last for 10-20 s.

In this study, MEG data were acquired in $650 \mathrm{~ms}$ time periods to better simulate real time activity. The time 


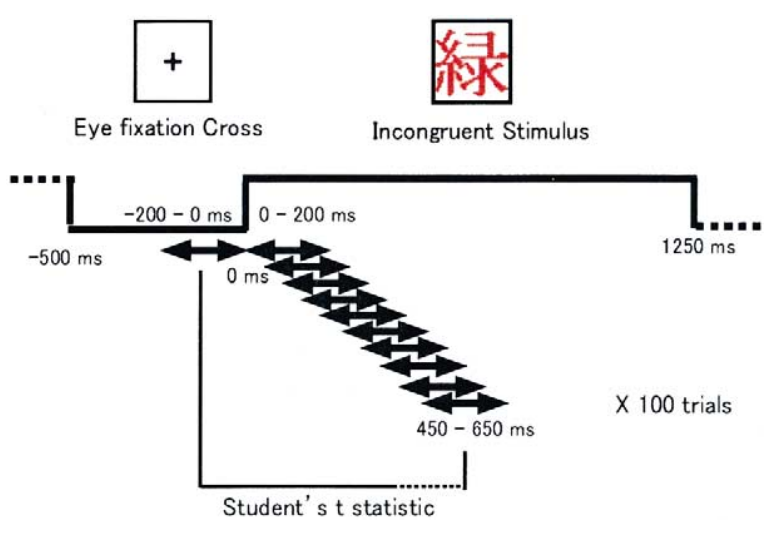

Fig. 1. CSD maps were estimated for each $200 \mathrm{~ms}$ time window with 100 incongruent stimulus data sets. The ten statistical imaging sets to demonstrate CSD changes from control to ten active states were produced with a sliding step of $50 \mathrm{~ms}$. In this example of incongruent stimulus, a Japanese kanji character representing "green" is printed in "red" ink.

sequence began with the introduction of the stimuli in the Stroop task, then subsequently analyzed using SAM with a $200 \mathrm{~ms}$ time window in $50 \mathrm{~ms}$ steps.

The subjects were five healthy right-handed [7] volunteers (22-27 years of age), whose native language was Japanese. Written consent was given by all subjects. The Stroop task required subjects to perform color naming instead of word reading. Incongruent stimuli, in which the word indicating the name of a color was written in a color different than that of the word (e.g., the word 'red' was printed in 'blue' ink), made subjects inhibit word reading. Twelve incongruent and four congruent stimuli using red, blue, yellow and blue colors were written in Japanese kanji characters. Each stimulus set consisted of an eye fixation period of $500 \mathrm{~ms}$ and an incongruent/congruent stimulus period of $1250 \mathrm{~ms}$ (Fig. 1). An eye fixation black cross and the visual stimuli were alternately presented on a 15 -inch TFT liquid crystal display placed $2 \mathrm{~m}$ away from the subjects. Incongruent and congruent stimulus sets were presented pseudorandomly to avoid habituation. Subjects were instructed to name the color quickly and accurately in a manner minimizing motion artifacts.

A helmet-shaped 64-channel superconducting quantum interference device (SQUID) sensor array (NeuroSQUID Model 100; CTF Systems Inc., Port Coquitlam, Canada) was used for the MEG measurements. MEG signals were digitized at $250 \mathrm{~Hz}$, filtered using a combined $60 \mathrm{~Hz}$ notch filter and $1-80 \mathrm{~Hz}$ band pass filter. The signals were recorded on a disk and analyzed off-line. One hundred unaveraged, incongruent stimulus sets without artifacts, were analyzed (Fig. 2).

Magnetic resonance (MR) images were obtained at $1.5 \mathrm{~T}$ (Magneton Impact; Siemens, Erlangen, Germany) with fiducial skin markers at the subject's nasion and bilateral preauricular points. Before and after MEG measurements, the position of the subject's head relative to the sensor array was measured using three small coils generating magnetic signals affixed to the same points. With these points, the images obtained from MEG could be superimposed on the subject's MR images.

The tomographic distributions of the activated regions were determined based on the unaveraged MEG measurements using a spatial filtering technique, SAM. Since MEG signals result from the spatial superposition of fields generated by neural source activity, spatial filtering is used to enhance the signal-to-noise ratios of spontaneous MEG by reduction of spatial superposition [11]. Using SAM, threedimensional images from current source density (CSD) mappings of MEG signal sources can be estimated. A statistical parametric map (SPM) can be produced using voxel-tovoxel comparison using the Student's $t$-test of the images taken in the control and active states. Functional images can be obtained by superimposing SPM on MR images $[3,16]$.

The $650 \mathrm{~ms}$ post-stimulus period was analyzed with a time window of $200 \mathrm{~ms}$ and a moving step of $50 \mathrm{~ms}$ for a total of ten active states. Reaction times ranging between $450-650 \mathrm{~ms}$ had been measured in three out of the five subjects with chin muscles electromyograms. The $200 \mathrm{~ms}$ period in which the subjects were looking at the eye fixation cross just before the presentation of the incongruent stimuli was regarded as the control state (Fig. 1). SPM was generated for five frequency bands with a $5 \mathrm{~mm}$ voxel resolution: $1-4,4-8,8-13,13-25$ and $25-60 \mathrm{~Hz}$. Images of cortical regions in which CSD significantly increased or decreased were examined. The reduction of CSD corresponded to ERD caused by the neural activation $[3,16]$.

In the $25-60 \mathrm{~Hz}$ band, SAM statistical imaging revealed significantly localized ERD. ERD began in the left parietaloccipital area (POA) at 150-250 ms post-stimulus; hereafter, the time given was the median of each time window. This was followed by the right prefrontal polar area (PPA) at

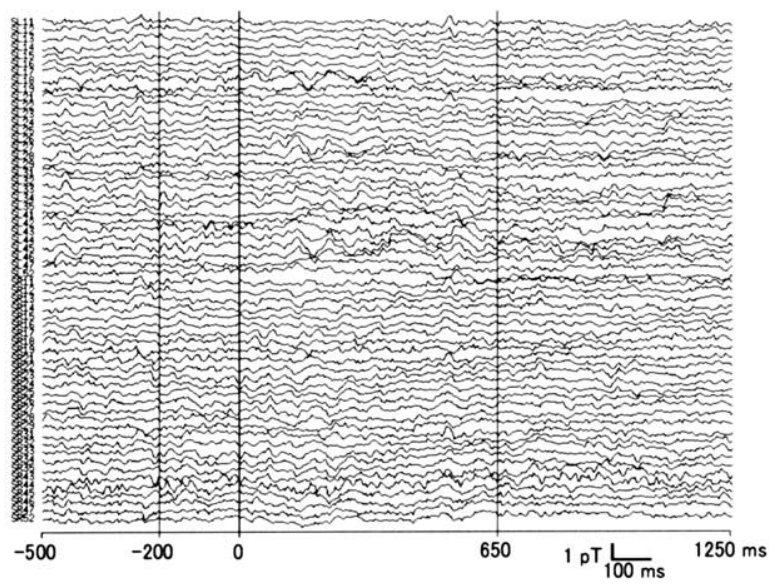

Fig. 2. An example of MEG signals in an incongruent stimulus set in Subject 1. One hundred epochs of MEG signals were filtered into five frequency bands and then submitted to SAM analysis. In this subject, signals from SL51, SR41, SR42 and SR51 were excluded from SAM analysis because of mechanical troubles of the sensors. Inspection of raw MEG signals cannot detect any MEG activity specific to the neural activation in the Stroop task. 


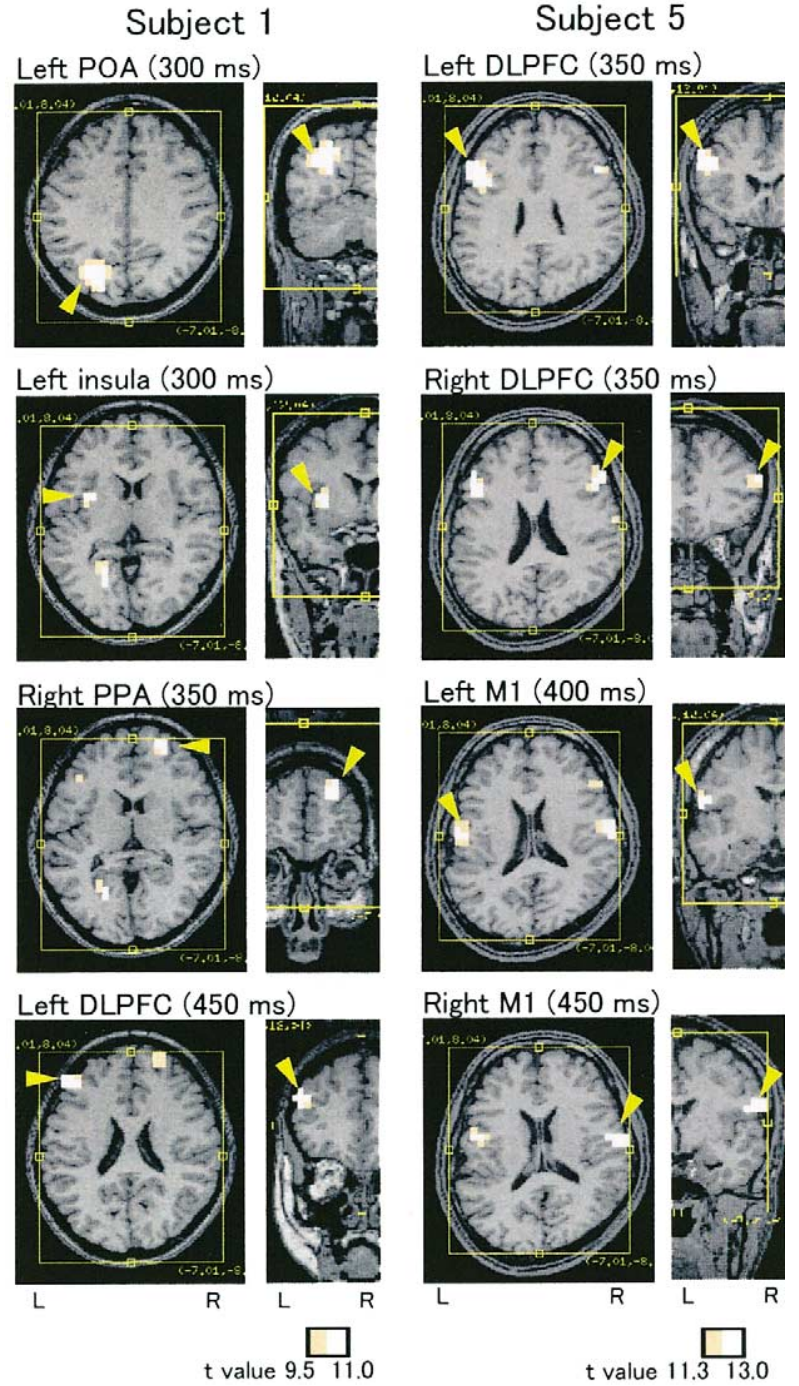

Fig. 3. The statistical imaging of CSD changes in the $25-60 \mathrm{~Hz}$ band during the Stroop task for Subject 1 and 5. In Subject 1, left POA, left insula, right PPA and left DLPFC showed significant CSD decrease (corresponding to neural activation) with its maximum $t$ value at $300 \mathrm{~ms}, 300 \mathrm{~ms}, 350 \mathrm{~ms}$ and $450 \mathrm{~ms}$ respectively. In Subject 5, left DLPFC (350 ms), right DLPFC (350 ms), left M1 $(400 \mathrm{~ms})$ and right M1 $(450 \mathrm{~ms})$ showed significant CSD decrease with maximum $t$ value. The voxels exhibiting statistically significant changes were color coded in red. Notice this MRI coordinate system is different from conventional ones. The horizontal slice is parallel to the plane including the nasion and bilateral preauricular points. The coronal slice is perpendicular to horizontal one. The time given is the median of each time window. The yellow arrows show the corresponding activated regions. POA, parietal-occipital area; PPA, prefrontal polar area; DLPFC, dorsolateral prefrontal cortex; M1, mid- to lower- primary motor area.

250-350 ms, the left DLPFC at $250-400 \mathrm{~ms}$, and the bilateral mid- to lower- primary motor areas (M1, cortical larynx area) at 350-400 ms with interindividual variability. Subsequently, ERD terminated at 300-400 ms, 400-500 ms, 350$550 \mathrm{~ms}$, and 400-550 ms, respectively (Figs. 3,4).

ERD in the left DLPFC was found in all five subjects, and that in the right in three. ERD in the left POA was found in three subjects, and that in the right PPA and the bilateral M1 in two subjects. The subjects were classified into two groups: three subjects (Subjects 1,2 and 3) showed ERD in POA without that in M1, and the other two (Subjects 4 and 5) showed ERD in M1 without that in POA (Fig. 4).

ERDs in the $1-4,4-8$, and $8-13 \mathrm{~Hz}$ bands did not show any common trends among the subjects. Although neuroimaging of the $13-25 \mathrm{~Hz}$ and the $25-60 \mathrm{~Hz}$ bands was similar, Student's $t$-test values of the $13-25 \mathrm{~Hz}$ band were smaller than those from the $25-60 \mathrm{~Hz}$ band. In the five frequency bands tested, no significant CSD decrease could be measured that corresponded to event-related synchronization.

The main result of this study was that of the four regions: the left POA, the right PPA, the left DLPFC, and bilateral $\mathrm{M} 1$, each were activated in a regular sequence with a time lag between 50 and $150 \mathrm{~ms}$. PDP from POA to M1 via PPA and DLPFC was visualized with temporal overlap.

The activation of the left DLPFC was observed in all five subjects, and that of the right in three subjects. Subjects were asked to inhibit word reading [6,14]. It had been reported that the performance of the Stroop task was reduced in prefrontal head-injured patients [9,15,17], suggesting the involvement of DLPFC in this inhibition. The go/no-go studies also reported that DLPFC was involved in inhibition. The no-go potential was observed on the dorsal bank of the principal sulcus of monkeys, which was equivalent to human DLPFC [12]. ECD was estimated in the dorsal part of bilateral frontal lobes at a latency of $135 \mathrm{~ms}$ in human MEG go/no-go studies [13].

The involvement of DLPFC had not been indicated by the

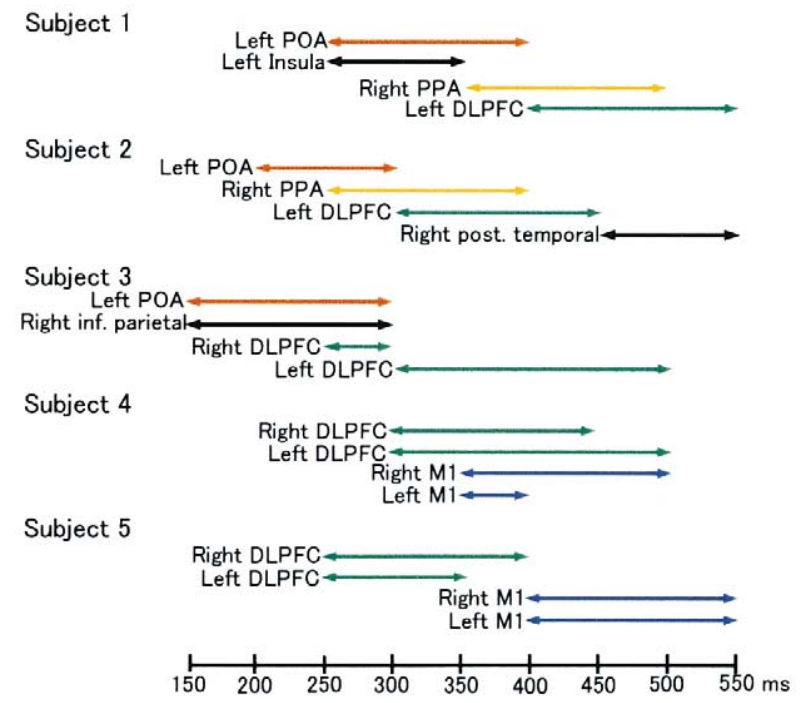

Fig. 4. Time course composite maps of activated regions during the Stroop task in all subjects. Thick line with arrows indicates significantly activated period. The time given is the median of each time window. POA, parietal-occipital area; PPA, prefrontal polar area; DLPFC, dorsolateral prefrontal cortex; M1, mid- to lower- primary motor area. 
block-designed PET [1,2,8] or fMRI studies [10], as was first reported using event-related fMRI with a time interval of $1.65 \mathrm{~s}$ [5]. This is probably due to the requirement of short time periods of 50-200 ms necessary to detect the transient activity in DLPFC. However, there were limitations even in this event-related fMRI method to estimate PDP in real time because of the time lag of 3-8 s between the actual activation and signal changes.

Activation in the right PPA was observed in two subjects. An fMRI study reported that bilateral fronto-polar prefrontal cortex was activated when subjects had to keep in mind a main goal while performing concurrent (sub) goal [4], indicating the allocation of attention. Since the main goal was color naming and the concurrent subgoal was inhibition of the habitual reaction of word reading, the activation in this area might be earlier than that in DLPFC.

The subjects were classified into two groups (Fig. 4). The regions related to visual information processing, allocation of attention and inhibition were activated in POA Dominant Group, while the regions related to inhibition and vocalization were activated in M1 Dominant Group. MEG SAM analysis enabled us to investigate inter-subject differences in the psychological strategy.

The activation of ACC was not observed in the present study. Presumably, SQUID sensors have difficulty in detecting magnetic fields from ACC, because: (1), Bilateral medial surfaces of ACC are positioned face to face and make cancellation of their magnetic fields; (2), The deeper location of ACC causes weaker MEG signals from reaching the sensors. Since PET, fMRI and MEG had advantages and disadvantages, these methods should complement each other in future studies.

[1] Bench, C.J., Frith, C.D., Grasby, P.M., Friston, K.J., Paulesu, E., Frackowiak, R.S.J. and Dolan, R.J., Investigations of the functional anatomy of attention using the Stroop test, Neuropsychologia, 31 (1993) 907-922.

[2] Carter, C.S., Mintum, M. and Cohen, J.D., Interference and facilitation effects during selective attention: $\mathrm{An} \mathrm{H}_{2}{ }^{15} \mathrm{O}$ PET study of Stroop task performance, Neuroimage, 2 (1995) 264-272.

[3] Ishii, R., Shinosaki, K., Ukai, S., Inouye, T., Ishihara, T., Yoshimine, T., Hirabuki, N., Asada, H., Kihara, T., Robinson, S.E. and Takeda, M., Medial prefrontal cortex generates frontal midline theta rhythm, Neuroreport, 10 (1999) 675679.
[4] Koechlin, E., Basso, G., Pietrini, P., Panzer, S. and Grafman, $\mathrm{J}$. , The role of the anterior prefrontal cortex in human cognition, Nature, 399 (1999) 148-151.

[5] Leung, H.C., Skudlarski, P., Gatenby, J.C., Peterson, B.S. and Gore, J.C., An event-related functional MRI study of the Stroop color word interference task, Cereb. Cortex, 10 (2000) 552-560.

[6] MacLeod, C.M., Half a century of research on the Stroop effect: a integrative review, Psychol. Bull., 109 (1991) 163203.

[7] Oldfield, R., The assessment and analysis of handedness: the Edinburgh Inventory, Neuropsychologia, 9 (1971) 97113.

[8] Pardo, J.V., Pardo, P.J., Janer, K.W. and Raicle, M.E., The anterior cingulate cortex mediates processing selection in the Stroop attentional conflict paradigm, Proc. Natl. Acad. Sci. USA, 87 (1990) 256-259.

[9] Perret, E., The left frontal lobe of man and the suppression of habitual response in verbal categorial befaviour, Neuropsychologia, 12 (1974) 323-330.

[10] Peterson, B.S., Skudlarski, P., Gatenby, J.C., Zhang, H.P., Anderson, A.W. and Gore, J.C., An fMRI study of Stroop word-color interference: evidence for cingulate subregions subserving multiple distributed attentional systems, Biol. Psychiatry, 45 (1999) 1237-1258.

[11] Robinson, S.E. and Rose, D.F., Current source image estimation by spatially filtered MEG, In M. Hoke, S.N. Eme and Y.C. Okada (Eds.), Biomagnetism: Clinical Aspects. Proceedings of the 8th International Conference on Biomagnetism, Elsevier, New York, 1992, pp. 761-765.

[12] Sasaki, K. and Gemba, H., Electrical activity in the prefrontal cortex specific to no-go reaction of conditioned hand movement with color discrimination in the monkey, Exp. Brain Res., 64 (1986) 603-606.

[13] Sasaki, K., Gemba, H., Nambu, A. and Matsuzaki, R., No-go activity in the frontal association cortex of human subjects, Neurosci. Res., 18 (1993) 249-252.

[14] Stroop, J.R., Studies of interference in serial verbal reactions, J. Exp. Psychol., 18 (1935) 643-662.

[15] Stuss, D.T., Floden, D., Alexander, M.P., Levine, B. and Katz, D., Stroop performance in focal lesion patients: dissociation of processes and frontal lobe lesion location, Neuropsychologia, 39 (2001) 771-786.

[16] Taniguchi, M., Kato, A., Fujita, N., Hirata, M., Tanaka, H., Kihara, T., Ninomiya, H., Hirabuki, N., Nakamura, H., Robinson, S.E., Cheyne, D. and Yoshimine, T., Movement-related desynchronization of the cerebral cortex studied with spatially filtered magnetoencephalography, Neuroimage, 12 (2000) 298-306.

[17] Vendrell, P., Junque, C., Pujol, J., Jurado, M.A., Molet, J. and Grafman, J., The role of prefrontal regions in the Stroop task, Neuropsychologia, 33 (1995) 341-352. 